\title{
Recent results from simulations of the magnetorotational instability
}

\author{
James M. Stone ${ }^{1}$ \\ ${ }^{1}$ Department of Astrophysical Sciences, Princeton University, Princeton NJ 08540, USA, \\ email: jmstone@princeton.edu
}

\begin{abstract}
The nonlinear saturation of the magnetorotational instability (MRI) is best studied through numerical MHD simulations. Recent results of simulations that adopt the local shearing box approximation, and fully global models that follow the entire disk, are described. Outstanding issues remain, such as a first-principles understanding of the dynamo processes that control saturation with no net magnetic flux. Important directions for future work include a better understanding of basic plasma processes, such as reconnection, dissipation, and particle acceleration, in the MHD turbulence driven by the MRI.
\end{abstract}

Keywords. accretion disks, instabilities, MHD, plasmas, turbulence, methods:numerical

\section{Introduction}

It has been nearly twenty years since Balbus \& Hawley (1991) identified and first recognized the importance of the magnetorotational instability (MRI) for angular momentum transport in accretion flows. It has been over 15 years since the first fully three dimensional MHD simulation of the nonlinear regime of the MRI (Hawley, Gammie \& Balbus 1995, hereafter HGB). In this time, there has been enormous progress in our understanding of the saturation mechanisms of the MRI, and at the same time new and puzzling questions have emerged that have yet to be understood. This paper provides a short review of some of those outstanding questions that have arisen in recent studies.

All of the work described here is based on numerical simulations using a variety of numerical algorithms as implemented in a variety of different codes. That such a wide range of codes have been used to study the MRI is important, because much greater confidence in a result is possible when it is confirmed by multiple workers using different methods. Many of the results I will present have been computed using a new Godunov method for MHD as implemented in the Athena code (Stone et al. 2008). Details of the numerical method required for studies of the MRI, for example the algorithms used for the shearing box source terms, and an orbital advection algorithm useful for global simulations, are described elsewhere (Stone \& Gardiner 2010).

\section{Local Shearing Box Simulations}

By expanding the MHD equations in a local frame corotating with the disk at some fiducial radius, it is possible to study the MRI in a local Cartesian coordinate system in a patch of the disk which is small compared to the radius (HGB). By focusing all of the computational resources on a small patch of the disk, much greater resolution can be afforded. Typically, the patch has dimensions of $H \times 4 H \times H$ in the radial, azimuthal, and vertical dimensions, where $H$ is the thermal scale height. More recently, orbital advection algorithms have allowed studies in much wider radial domains, by eliminating 

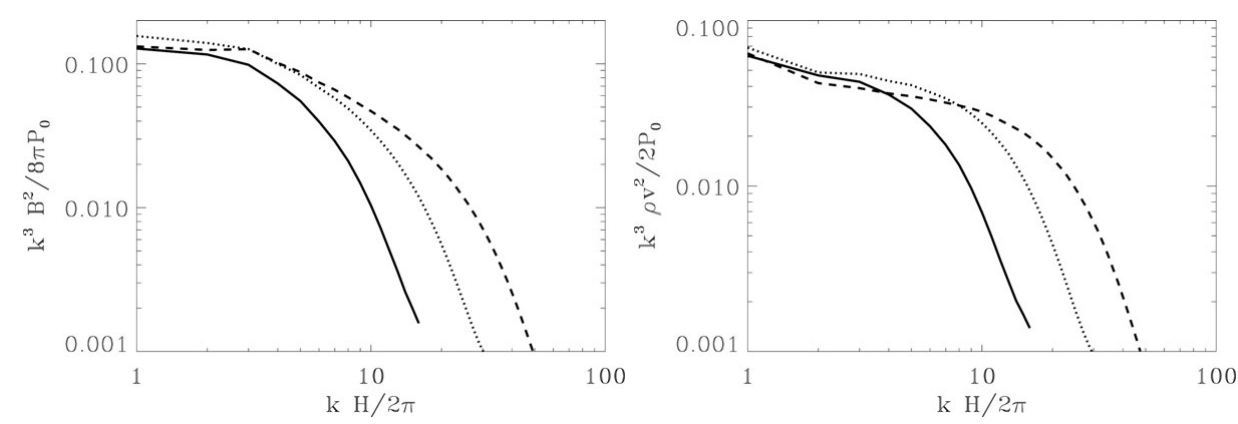

Figure 1. Power spectrum of the magnetic energy (left) and kinetic energy (right) averaged over orbits 50-100 in unstratified shearing box simulations of the MRI with net flux and no explicit dissipation. The solid, dotted, and dashed lines correspond to resolutions of $32 / H, 64 / H$, and $128 / H$ respectively.
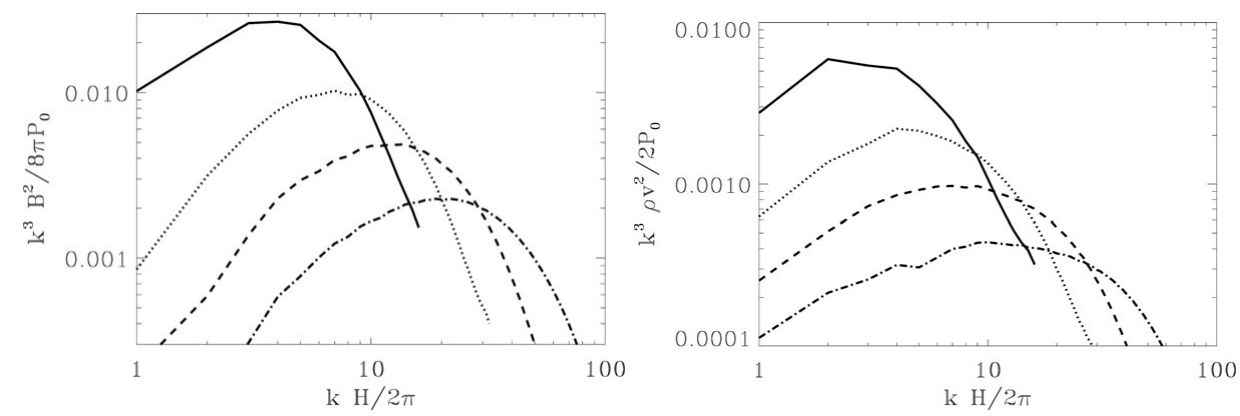

Figure 2. Same as Figure 1, but for the case of no net flux and resolutions up to $256 / \mathrm{H}$.

the background azimuthal flow from the stability constraint in the time step. Here we summarize results from two kinds of local, shearing box studies.

\section{Unstratified Disks.}

If the vertical extent of the domain is small, then the vertical component of gravity can be neglected, and there is no stratification of the density. Such models are appropriate for the midplane of the disk.

When the simulation domain contains net vertical flux of magnetic field, then HGB found the saturation amplitude increases with increasing flux. More recent studies have confirmed this result, and have also explored the dependence of the saturation amplitude on other parameters (Sano et al. 2003). Convergence of the stress is observed at very modest resolutions, about $32 / H$. Increasing the radial extent of the domain decreases fluctuations in the stress caused by channel solutions but does not change the timeaveraged value of the stress (Bodo et al. 2008). Figure 1 shows the Fourier power spectrum of the kinetic and magnetic energies at different resolutions in simulations with net vertical flux characterized by an initial $\beta=1600$. Note that the power spectrum looks very similar to classical turbulence, with most of the power at small $k$, decreasing in a power law until the dissipation scale is reached.

One of the most important recent results was reported by Fromang \& Papaloizou (2007) who found that the saturation level of the stress in unstratified disks with no-net flux and no explicit dissipation decreases as the resolution is increased. Figure 2 shows the Fourier power spectrum of the kinetic and magnetic energies at different resolutions simulations with no net flux. Note the results are vastly different from the net flux case (Figure 1). Now most of the power is at some intermediate wavenumber which changes 
with resolution, and the decrease in the total power is clearly evident as the resolution is increased.

In another important paper, Fromang et al. (2007) reported that the saturation amplitude with no net flux is constant with numerical resolution once explicit dissipation (viscosity and resistivity) is added. The interpretation is that fixing the dissipation fixes the magnetic Prandtl number $P_{\mathrm{m}}=\nu / \eta$, where $\nu$ and $\eta$ are the coefficients of viscosity and resistivity respectively, and that a converged solution is possible only at fixed $P_{\mathrm{m}}$ and Reynolds numbers. When only the grid provides dissipation, changing the resolution can change the effective Reynolds number, and there is no converged solution. In fact, Fromang et al. (2007) also found that sustained turbulence is not possible if the Reynolds number is too low.

It is interesting to note that a dynamo must be at work to explain the sustained turbulence observed in zero net flux simulations. The fact that the amplitude of the stress decreases with increasing resolution when there is no explicit dissipation, but that turbulence is still sustained, suggests that the dynamo is sensitive to the range of scales set by the box size and grid resolution. Recently Yousef et al. (2008) have reported that shear dynamos driven by turbulence are sensitive to the vertical box size. Is this true for the MRI? Work by Lesur \& Ogilvie (2008) confirm that the saturation amplitude of the MRI with no net flux, and explicit dissipation, is increased when the vertical box size is increased. Recently we have found the same result even with no explicit dissipation. By increasing the box size in the vertical dimension to $2 H$ we observe much larger amplitude stress in the saturated state. Even more remarkable is that this stress is independent of resolution even with no explicit dissipation, as shown in Figure 3. Clearly much work remains to understand the properties of the dynamo with no explicit dissipation.

Finally, there has been substantial effort to understand the effect of explicit dissipation on the MRI with net vertical flux. At low Reynolds numbers, Lesur \& Longaretti (2007) found the stress was very strongly dependent on the Reynolds number. More recent studies of net toroidal fields by Simon \& Hawley (2009) confirm this result, although they also suggest the Prandtl number dependence may be decreasing as the Reynolds number is increased. Such studies are challenging since very high resolutions are required to study the high Reynolds number regime, but there continues to be much effort in this direction.

\section{Density stratified Disks.}

Using the local shearing box, it is also possible to study the saturation of the MRI including the effect of vertical gravity, which produces stratification of the density. Early work (e.g. Miller \& Stone 2000) showed that buoyancy produced a magnetized corona above the surface of the disk, and that magnetic energy rises from the midplane in a quasiperiodic fashion. New simulations are able to evolve stratified disks for much longer, with higher resolution, making these processes much clearer.

Figure 4 shows a space-time plot of the radial, azimuthal, and vertical components of the magnetic field ( $B_{x}, B_{y}$, and $B_{z}$ respectively), as well as the $x-y$ component of the Maxwell stress. These quantities are computed by averaging each variable over the $x-$ and $y$-directions, and then plotting the resulting vertical variation versus time. The calculation has no net flux, and no explicit dissipation.

Note the striking "butterfly" pattern evident in $B_{y}$. Clearly the dynamo at the midplane is creating toroidal field, which rises buoyantly out of the disk. Recently Gressel (2010) has modeled this dynamo using mean field theory and shown remarkable agreement between the spacetime plots of the model and simulations.

Also of interest are the time evolution of the stress as a function of resolution. Once again, the same level of stress is achieved, regardless of numerical resolution (Davis 


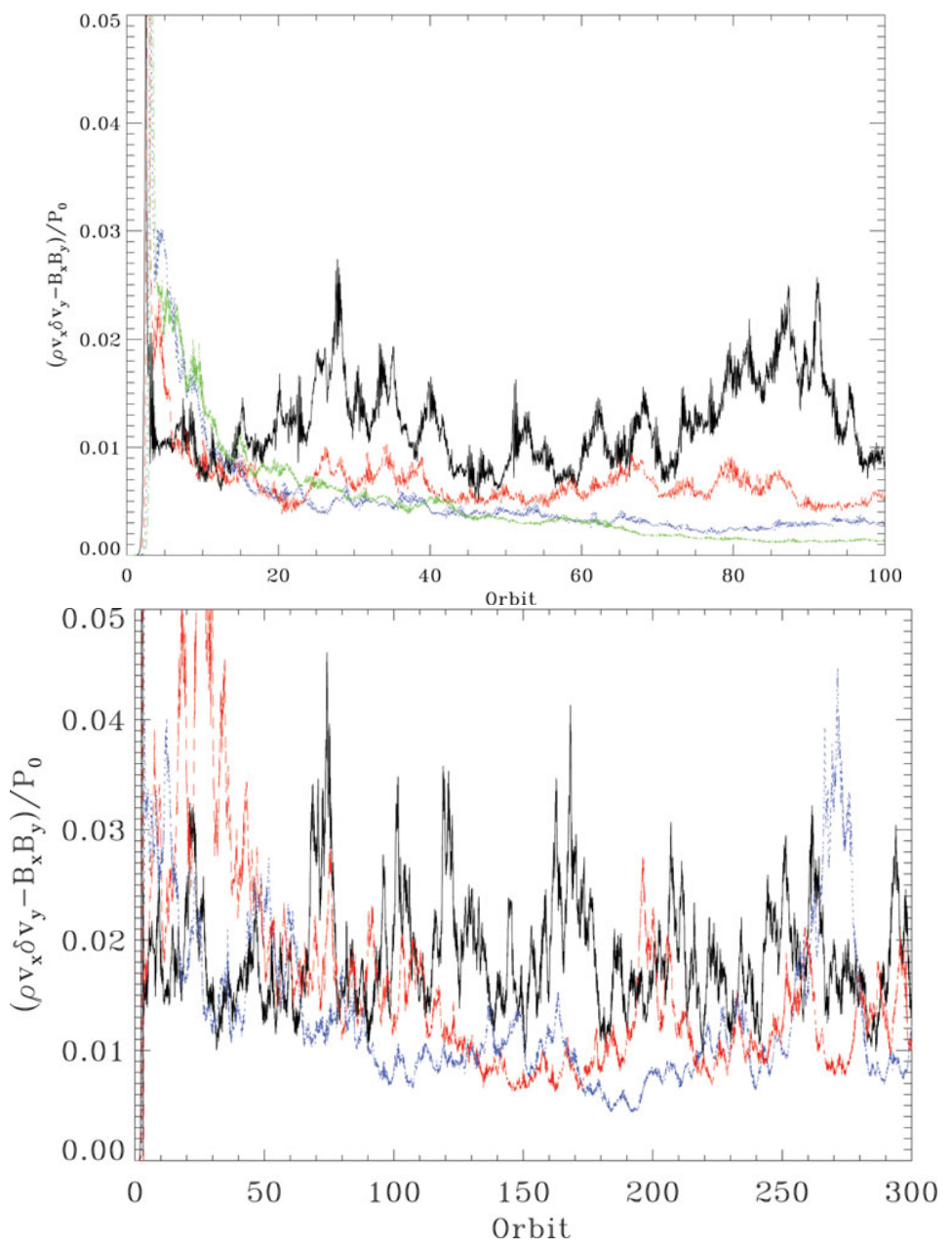

Figure 3. Time evolution of the stress at resolutions of $32 / H$ (black), $64 / H$ (red), 128/H (blue), and $256 / H$ (green) for unstratified shearing box simulations of the MRI with no net flux and no explicit dissipation. The top panel is in a domain of $H \times 4 H \times H$, while in the bottom the vertical box size is increased to $2 H$. Note there is convergence in the latter.

et al. 2010; Shi \& Krolik 2010). Stratified disks simulations have a vertical extent much larger than $H$, thus the fact the stress converges with resolution is in agreement with the result shown above for unstratified simulations with no net flux and no explicit dissipation in domains with large vertical extent. Davis et al. (2010) also show that sustained turbulence is achieved in stratified disks even at Reynolds numbers where the MRI is suppressed in unstratified disks. Taken together, these results demonstrate the importance of understanding the dynamo in the case of no net flux, and no explicit dissipation.

Finally, it is worth commenting that although the zero net flux case is of interest as an idealized model, it probably has no relevance for real disks. In the shearing box approximation, if there is no net flux initially, then there can never be any net flux at any time in the evolution. That an accretion disk could organize itself such that every $H \times 4 H \times H$ domain in the disk had exactly no net flux for all time seems impossible. 
Still, understanding the dynamics in the no net flux simulations could provide important insights to real disks.

\section{Global Simulations}

In addition to shearing box simulations, global simulations of the MRI in disks have been performed by many authors, for example Armitage (1998) and Hawley (2000) studied the saturation of the MRI in cylindrical disks with no stratification. Space limitations prevent a proper review of all we have learned about the MRI from global simulations here. Instead, one new direction being followed by several groups will be mentioned.

The dramatic increase in computational resources, combined with better algorithms (such as orbital advection to increase the timestep) means that very high resolution simulations of global disks can be performed. Here, high resolution means the same number of grid points per thermal scale height is the same as commonly used for local shearing box simulations. Figure 5 shows an image of the density from such a calculation, performed by K. Sorathia and C. Reynolds. Their study uses resolutions from $8 / H$ up
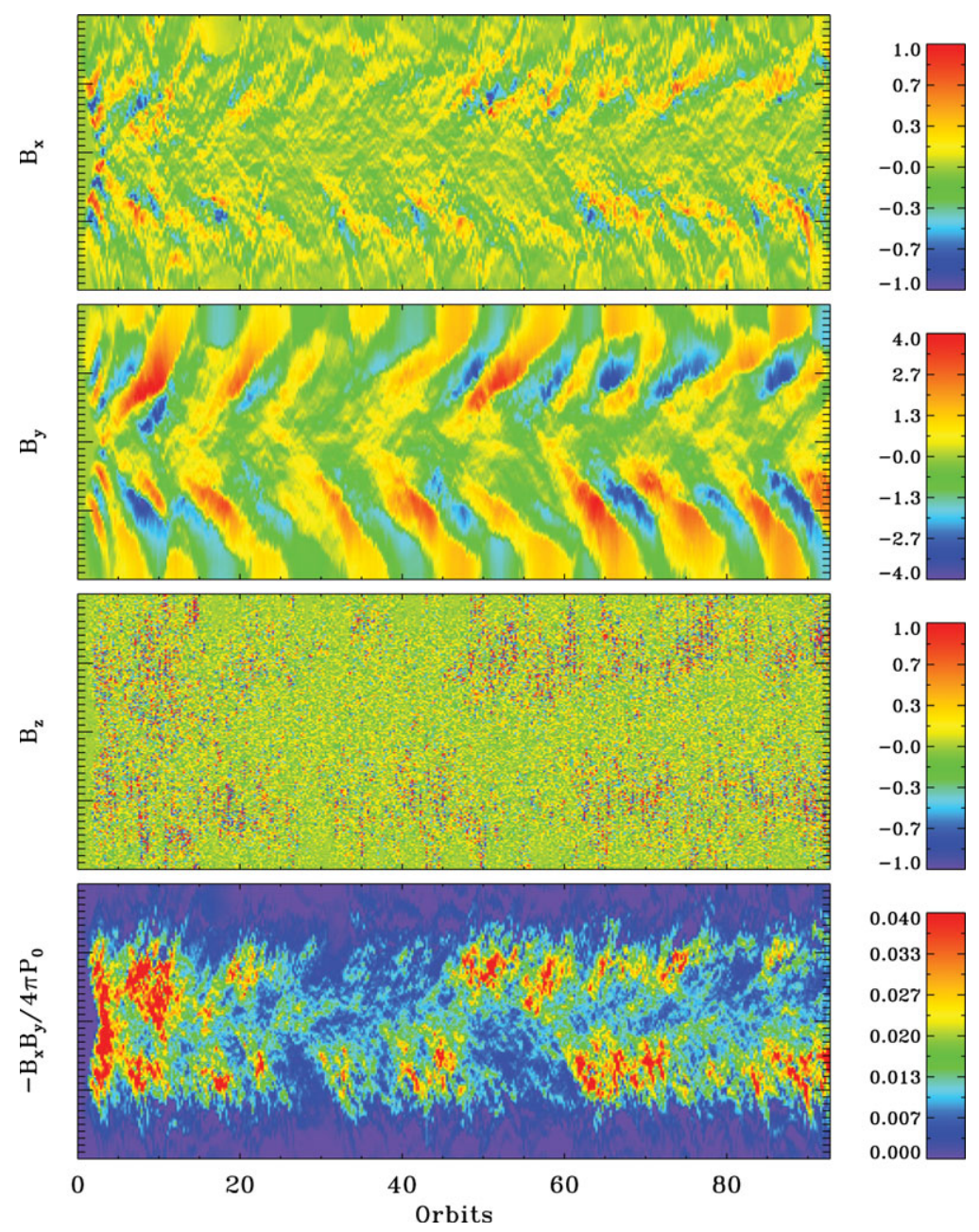

Figure 4. Space-time plots of various quantities in a density stratified shearing box simulation of the MRI. 


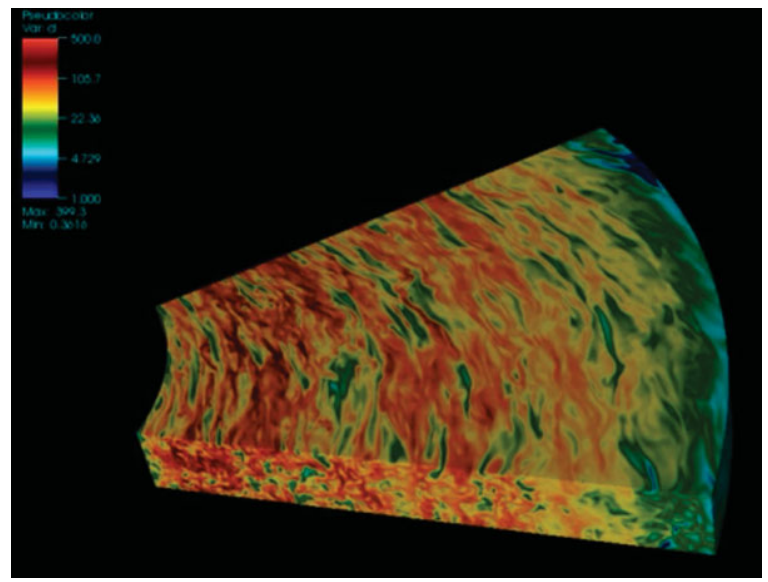

Figure 5. Image of the density after 30 orbits at the inner edge in a global cylindrical disk simulations of the MRI with a resolution of $16 / H$.

to $32 / H$ over the entire disk. By extracting small volumes equivalent to local shearing boxes, direct comparison can be made to previous work. Although this work is ongoing, an early result is that although the simulation begins with no net flux within each local volume, it quickly evolves to a state where there is a distribution of net fluxes, confirming the expectation above.

\section{Summary and Future Work}

At a meeting on plasma physics, it seems appropriate to focus on properties of the MRI that are connected to fundamental plasma processes. Recently, the properties of the MRI in weakly collisional plasmas (in the limit where the mean free path is large compared to the ion gyroradius) have begun to be explored. In the Braginskii regime, where kinetic effects are included through an anisotropic viscosity, it has been shown the growth rate of the MRI at long wavelengths for very weak fields is enhanced enormously (Quataert, Dorland, \& Hammett 2002; Sharma, Hammett, \& Quataert 2003; Islam \& Balbus 2005). New simulations using the Braginskii equations of MHD have begun to explore this regime, which could be important for magnetic field amplification in protogalaxies.

A more sophisticated treatment of the kinetic plasma effects on the MRI was presented by Sharma et al. (2006). They found that at saturation, there was significant angular momentum transport by anisotropic pressure. In a two fluid study (with both electrons and ions) they found that anisotropic pressure was also responsible for significant heating of the electrons (Sharma et al. 2007), which has significant implications for weakly collisional accretion flows around compact objects. These studies have required an $a d$ hoc treatment of micro-instabilities such as firehose and mirror modes, and much remains to be done to understand the effect of these on the MHD turbulence driven by the MRI in the kinetic regime. Ultimately, full PIC simulations of the MRI could settle important questions, like whether the combination of turbulence driven by the MRI and shear can accelerate particles, and whether energy dissipation occurs primarily in the ions or electrons, or equally in both.

In summary, although tremendous progress has been made towards understanding of angular momentum transport in accretion disks by the MRI using MHD simulations, much work remains to be done. For example, the properties of the dynamo driven by the MRI in the case of no net flux, as well as the butterfly diagram observed in stratified 
disks, remain to be fully understood. Fundamental astrophysics questions remain, such as what causes quasi-periodic oscillations in X-ray binaries. Finally, as discussed above, fundamental plasma problems remain.

\section{References}

Armitage, P. J. 1998, ApJ, 501, L189

Balbus, S. A. \& Hawley, J. F. 1991, ApJ, 376, 214

Bodo, G., Mignone, A., Cattaneo, F., Rossi, P., \& Ferrari, A. 2008, A\&A A, 487, 1

Davis, S. W., Stone, J. M., \& Pessah, M. 2010, ApJ 713, 52

Fromang, S. \& Papaloizou, J. 2007, A\& A, 468, 1

Fromang, S., Papaloizou, J., Lesur, G., \& Heinemann, T. 2007, A\&A, 476, 1123

Gressel, O. 2010, MNRAS, 405, 41

Hawley, J. F., Gammie, C. F., \& Balbus, S. A. 1995, ApJ 440, 742

Hawley, J. F. 2001, ApJ 554, 534

Islam, T. S. \& Balbus, S. A. 2005, ApJ 633, 328

Lesur, G. \& Longaretti, P.-Y. 2007, A\&A 378, 1471

Lesur, G. \& Ogilvie, G. I. 2008, $A \& A 488,451$

Miller, K. A. \& Stone, J. M. 2000, ApJ 534, 398

Quataert, E., Dorland, W., \& Hammett, G. W. 2002, ApJ 577, 524

Sano, T., Inutsuka, S., Turner, N., \& Stone, J. M. 2004, ApJ 605, 321

Sharma, P., Hammett, G. W., \& Quataert, E. 2003, ApJ 596, 1121

Sharma, P., Hammett, G. W., Quataert, E., \& Stone, J. M. 2006, ApJ 637, 952

Sharma, P., Hammett, G. W., Quataert, E., \& Stone, J. M. 2007, ApJ 667, 714

Shi, J., Krolike, J., \& Hirose, S. 2010, ApJ 708, 1716

Simon, J. B. \& Hawley, J. F. 2009, ApJ 707, 833

Stone, J. M., Hawley, J. F., Gammie, C. F., \& Balbus, S. A. 2010, ApJS, 178, 137

Stone, J. M. \& Gardiner, T. A. 2010, ApJS, 189, 142

Yousef, T. A., et al. 2008, Phs. Rev. Lett., 100, 4501 